\title{
Derivatives of the Grüneisen and Einstein Functions
}

\author{
Ared Cezairliyan \\ Institute for Materials Research, National Bureau of Standards, Washington, D.C. 20234
}

(May 13, 1970)

\begin{abstract}
Expressions (in closed form) are derived for the first five derivatives of the Grüneisen and Einstein functions. Recursion formulas for the successive derivatives of both functions are also given. Computations are made and tabular results are given for the first five derivatives of the Grüneisen and Einstein functions.
\end{abstract}

Key words: Einstein function; electrical resistivity; Grüneisen function; solid state physics; transport properties.

\section{Introduction}

According to the solid state theory of matter, the mathematical expression for the electrical resistivity $\rho$, of good conductors is (ref. [1] ${ }^{1}$ )

$$
\rho=\frac{A T}{\theta}\left[G^{0}\left(\frac{\theta}{T}\right)\right]
$$

where,

$$
\begin{aligned}
A & =\text { constant } \\
\theta & =\text { Debye temperature } \\
T & =\text { absolute temperature } \\
G^{0}\left(\frac{\theta}{T}\right) & =4\left(\frac{T}{\theta}\right)^{4} \int_{0}^{\theta / T} \frac{\zeta^{5} d \zeta}{\left(e^{\zeta}-1\right)\left(1-e^{-\zeta}\right)} .
\end{aligned}
$$

The function $G^{0}(\theta / T)$ is frequently referred to as the Grüneisen function. This function is approximately unity for $T \gg \theta$, which indicates that at high temperatures electrical resistivity increases linearly with temperature. At very low temperatures where $T \ll \theta$, the Grüneisen function can be expressed approximately by $G^{0}(\theta / T) \approx B(T / \theta)^{4}$, which indicates that at very low temperatures electrical resistivity increases with the fifth power of temperature.

For various calculations, the successive derivatives of the electrical resistivity function may be needed. This requires a knowledge of the derivatives of the Grüneisen function. Also, it may be possible to make further refinements in the electrical resistivity expression by expanding the resistivity function in the derivatives of the Grüneisen function. It was observed that the derivatives of the Grüneisen function contain the Einstein function and its successive derivatives. The objective of this writing is to obtain expressions for the successive derivatives of the Grüneisen and the Einstein functions. 


\section{Derivatives of the Gruneisen Function}

Defining $x=\theta / T$, eq (2) becomes

$$
G^{0}(x)=\frac{4}{x^{4}} \int_{0}^{x} \frac{\zeta^{5} d \zeta}{\left(e^{\zeta}-1\right)\left(1-e^{-\zeta}\right)}
$$

Differentiating eq (3) and simplifying the result, one obtains

$$
\frac{d}{d x}\left[G^{0}(x)\right]=G^{1}(x)=-\frac{4}{x}\left[\frac{4}{x^{4}} \int_{0}^{x} \frac{\zeta^{5} d \zeta}{\left(e^{\zeta}-1\right)\left(1-e^{-\zeta}\right)}\right]+\frac{4}{x}\left[\frac{x^{2} e^{-x}}{\left(1-e^{-x}\right)^{2}}\right] .
$$

The Einstein function is defined as

$$
E^{0}(x)=\frac{x^{2} e^{-x}}{\left(1-e^{-x}\right)^{2}}
$$

Substitution of eqs (3) and (5) in eq (4) yields

$$
G^{1}(x)=-\frac{4}{x}\left[G^{0}(x)-E^{0}(x)\right]
$$

For convenience, the variable $x$ in the functions $G^{0}(x), E^{0}(x)$, and in their derivatives $G^{1}(x)$, $E^{1}(x)$, etc. will be omitted and will be written simply as $G^{0}, E^{0}, G^{1}, E^{1}$, etc., respectively. One may note that the superscript numerals on both $G$ and $E$ indicate the order of differentiation.

The following is a list of the first five derivatives of the Grüneisen function obtained by the successive differentiation of eq (3) expressed in terms of the lower order derivatives of the Grüneisen and Einstein functions.

$$
\begin{aligned}
G^{1} & =-\frac{4}{x} G^{0}+\frac{4}{x} E^{0} \\
G^{2} & =-\frac{5}{x} G^{1}+\frac{4}{x} E^{1} \\
G^{3} & =-\frac{6}{x} G^{2}+\frac{4}{x} E^{2} \\
G^{4} & =-\frac{7}{x} G^{3}+\frac{4}{x} E^{3} \\
G^{5} & =-\frac{8}{x} G^{4}+\frac{4}{x} E^{4}
\end{aligned}
$$

The recursion formula for the above is

$$
G^{n}=-\left(\frac{n+3}{x}\right) G^{n-1}+\frac{4}{x} E^{n-1}
$$

Where, $n$ is the order of differentiation. It may be seen that the $n$th derivative of the Grüneisen function is given in terms of its $(n-1)$ th derivative. In order to be able to express the $n$th derivative of the Grüneisen function in terms of the function itself, successive substitutions for the lower order derivatives are made. 
The following is a list of the first five derivatives of the Grüneisen function in terms of the Grüneisen function and the derivatives of the Einstein function.

$$
\begin{aligned}
& G^{1}=-\frac{4}{x}\left(G^{0}-E^{0}\right) \\
& G^{2}=\frac{20}{x^{2}}\left(G^{0}-E^{0}\right)+\frac{4}{x} E^{1} \\
& G^{3}=-\frac{120}{x^{3}}\left(G^{0}-E^{0}\right)-\frac{24}{x^{2}} E^{1}+\frac{4}{x} E^{2} \\
& G^{4}=\frac{840}{x^{4}}\left(G^{0}-E^{0}\right)+\frac{168}{x^{3}} E^{1}-\frac{28}{x^{2}} E^{2}+\frac{4}{x} E^{3} \\
& G^{5}=-\frac{6720}{x^{5}}\left(G^{0}-E^{0}\right)-\frac{1344}{x^{4}} E^{1}+\frac{224}{x^{3}} E^{2}-\frac{32}{x^{2}} E^{3}+\frac{4}{x} E^{4}
\end{aligned}
$$

The recursion formula for the above is

$G^{n}=\frac{S_{n 1} C_{n 1}}{x^{n}}\left(G^{0}-E^{0}\right)+\frac{S_{n 2} C_{n 2}}{x^{n-1}} E^{1}+\frac{S_{n 3} C_{n 3}}{x^{n-2}} E^{2}+\ldots \quad+\frac{S_{n i} C_{n i}}{x^{n-i+1}} E^{i-1}+\ldots+\frac{S_{n n} C_{n n}}{x} E^{n-1}$

where, $C_{n i}$ and $S_{n i}$ indicate the absolute value and the sign of the coefficients, respectively, and are expressed by the following relations.

$$
\begin{array}{ll}
C_{11}=4 & \\
C_{n i}=0 & {[i>n]} \\
C_{n 1}=(n+3) C_{n-1,1} & {[n \geqslant 2]} \\
C_{n i}=(n-i) C_{n-1, i}+C_{n-1, i-1} & {[n \geqslant i \geqslant 2]} \\
S_{n 1}=(-1)^{n} & \\
S_{n i}=(-1)^{n+i} & {[n \geqslant i \geqslant 2]}
\end{array}
$$

The numerical values of the product $S_{n i} C_{n i}$ required for the first seven derivatives of the Grüneisen function are given in table 1 .

It can be seen from eq (18) that the relation for the derivative of the Grüneisen function contains the derivatives of the Einstein function. The latter are obtained in the next section.

\section{Derivatives of the Einstein Function}

The Einstein function is defined by eq (5). For mathematical convenience, it may be written as

$$
E^{0}=\left(\frac{x^{2}}{1-e^{-x}}\right) y
$$


TABLE 1. List of the coefficients $\mathrm{S}_{\mathrm{ni}} \mathrm{C}_{\mathrm{ni}}$ that appear in eq (18)

\begin{tabular}{|c|c|c|c|c|c|c|c|}
\hline \multirow[b]{2}{*}{$S_{n i} C_{n i}$} & \multicolumn{7}{|c|}{$i \rightarrow$} \\
\hline & 1 & 2 & 3 & 4 & 5 & 6 & 7 \\
\hline 1 & -4 & 0 & 0 & 0 & 0 & 0 & 0 \\
\hline 2 & 20 & 4 & 0 & 0 & 0 & 0 & 0 \\
\hline 3 & -120 & -24 & 4 & 0 & 0 & 0 & 0 \\
\hline 4 & 840 & 168 & -28 & 4 & 0 & 0 & 0 \\
\hline 5 & $-6,720$ & $-1,344$ & 224 & -32 & 4 & 0 & 0 \\
\hline 6 & 60,480 & 12,096 & $-2,016$ & 288 & -36 & 4 & 0 \\
\hline 7 & $-604,800$ & $-120,960$ & $20 ; 160$ & $-2,880$ & 360 & -40 & 4 \\
\hline
\end{tabular}

where,

$$
y=\frac{e^{-x}}{1-e^{-x}}=\frac{1}{e^{x}-1} .
$$

Differentiation of eq (20) and simplification yields

$$
\frac{d E^{0}}{d x}=E^{1}=2 D^{0} E^{0}
$$

where,

$$
D^{0}=-\frac{1}{2}+\frac{1}{x}-y \text {. }
$$

The second derivative of $E^{0}$ becomes

$$
\frac{d E^{1}}{d x}=E^{2}=2 D^{0} E^{1}+2 D^{1} E^{0}
$$

where,

$$
\mathrm{D}^{1}=\frac{d D^{0}}{d x}=-\frac{1}{x^{2}}+y+y^{2}
$$

It should be noted that the superscript numeral of $D$ indicates the order of differentiation similar to those used for $G$ and $E$. It may be seen that the derivatives of the Einstein function contain the derivatives of the quantity $y^{m}$ for various values of $m$, where $m$ indicates the power of $y$. The recursion formula for the derivatives of $y^{m}$ is

$$
\frac{d}{d x}\left(y^{m}\right)=-m y^{m}(y+1) .
$$

The following is a list of the first five derivatives of the Einstein function obtained by the successive differentiation of eq (20).

$$
\begin{aligned}
& E^{1}=2 D^{0} E^{0} \\
& E^{2}=2 D^{0} E^{1}+2 D^{1} E^{0} \\
& E^{3}=2 D^{0} E^{2}+4 D^{1} E^{1}+2 D^{2} E^{0}
\end{aligned}
$$




$$
\begin{aligned}
& E^{4}=2 D^{0} E^{3}+6 D^{1} E^{2}+6 D^{2} E^{1}+2 D^{3} E^{0} \\
& E^{5}=2 D^{0} E^{4}+8 D^{1} E^{3}+12 D^{2} E^{2}+8 D^{3} E^{1}+2 D^{4} E^{0}
\end{aligned}
$$

The recursion formula for the above is

$E^{n}=K_{n 1} D^{0} E^{n-1}+K_{n 2} D^{1} E^{n-2}+K_{n 3} D^{2} E^{n-3}+\ldots+K_{n i} D^{m-1} E^{n-i}+\ldots+K_{n n} D^{n-1} E^{0}$

where, $n$ indicates the order of differentiation.

$K_{n i}$ are constants and can be obtained from the following relations

$$
\left.\begin{array}{ll}
K_{n 1}=2 & \\
K_{n i}=0 & {[i>n]} \\
K_{n i}=K_{n-1, i}+K_{n-1, i-1} . & {[n \geqslant i \geqslant 2]}
\end{array}\right\}
$$

$D^{m}$ are coefficients which are functions of $x$. The superscript $m$ indicates the order of differentiation. They are expressed by the following equations.

$D^{0}=-\frac{1}{2}+\frac{1}{x}-y$

$D^{m}=(-1)^{m+1}[-\frac{m !}{x^{m+1}}+\kappa_{m 1} y+\kappa_{m 2} y^{2}+\kappa_{m 3} y^{3}+\ldots+\kappa_{m j} y^{j}+\ldots+\kappa_{m, m+1} y^{m+1} \cdot \underbrace{}_{[m \geqslant 1]}$

where,

\begin{tabular}{|c|c|c|c|c|c|c|c|}
\hline \multirow[b]{2}{*}{$K_{n i}$} & \multicolumn{7}{|c|}{$i \rightarrow$} \\
\hline & 1 & 2 & 3 & 4 & 5 & 6 & 7 \\
\hline 1 & 2 & 0 & 0 & 0 & 0 & 0 & 0 \\
\hline 2 & 2 & 2 & 0 & 0 & 0 & 0 & 0 \\
\hline$n \quad 3$ & 2 & 4 & 2 & 0 & 0 & 0 & 0 \\
\hline$\downarrow \quad 4$ & 2 & 6 & 6 & 2 & 0 & 0 & 0 \\
\hline 5 & 2 & 8 & 12 & 8 & 2 & 0 & 0 \\
\hline 6 & 2 & 10 & 20 & 20 & 10 & 2 & 0 \\
\hline 7 & 2 & 12 & 30 & 40 & 30 & 12 & 2 \\
\hline
\end{tabular}

$$
\begin{aligned}
& \kappa_{m 1}=\kappa_{12}=1 \\
& \kappa_{m j}=0 \quad[j>(m+1)] \\
& \kappa_{m j}=j\left(\kappa_{m-1, j}\right)+(j-1)\left(\kappa_{m-1, j-1}\right) \quad[j \geqslant 2, m \geqslant 2, j \leqslant(m+1)] .
\end{aligned}
$$

\begin{tabular}{|c|c|c|c|c|c|c|c|c|c|}
\hline \multicolumn{2}{|c|}{$\kappa_{m j}$} & \multicolumn{8}{|c|}{$j \rightarrow$} \\
\hline & 1 & 1 & 1 & 0 & 0 & 0 & 0 & 0 & 0 \\
\hline & 2 & 1 & 3 & 2 & 0 & 0 & 0 & 0 & 0 \\
\hline$m$ & 3 & 1 & 7 & 12 & 6 & 0 & 0 & 0 & 0 \\
\hline$\downarrow$ & 4 & 1 & 15 & 50 & 60 & 24 & 0 & 0 & 0 \\
\hline & 5 & 1 & 31 & 180 & 390 & 360 & 120 & 0 & 0 \\
\hline & 6 & 1 & 63 & 602 & 2,100 & 3,360 & 2,520 & 720 & 0 \\
\hline & 7 & 1 & 127 & 1,932 & 10,206 & 25,200 & 31,920 & 20,160 & 5,040 \\
\hline
\end{tabular}

The coefficients $K_{n i}$ and $\kappa_{m j}$ can be represented as matrix elements. Their numerical values, required for the first seven derivatives of the Einstein function, are given in tables 2 and 3 , respectively.

TABLE 2. List of the coefficients $\mathrm{K}_{\mathrm{ni}}$ that appear in eq (32)

TABLE 3. List of the coefficients $\kappa_{\mathrm{mj}}$ that appear in eq (35) 
Calculating the derivatives of the Einstein function using eq (32) and substituting these in either eq (12) or eq (18) one can obtain the derivatives of the Grüneisen function. Since the $n$th derivative of the Grüneisen function contains all lower derivatives (up to $n-1$ ) of the Einstein function, it is sufficient to derive a recursion formula for the derivatives of the Einstein function in terms of its lower derivatives. However, in the case of the Grüneisen function, a recursion formula for its derivatives was obtained both in terms of lower derivatives of $G$ and in terms of $G$ itself.

\section{Computation of the Derivatives of the Einstein and Grüneisen Functions}

Based on the formulas developed in the previous sections, the first five derivatives of the Einstein and Grüneisen functions are computed for the range $1<x<30$ using a computer. The selection of the limits is that of a practical one. Above the Debye temperature $(x=1)$ both functions are relatively insensitive to temperature variations and approach unity as temperature increases. Below $T=\theta / 30(x=30)$ the values of the functions become small and also the integral in the Grüneisen function attains a constant value.

The Einstein function and its first five derivatives are computed using eqs (20), (27), (28), (29), (30), and (31), respectively in conjunction with eqs (34), (35), and (36). The results are given in table 4. The values obtained for the Einstein function are in exact agreement with those reported by Hilsenrath and Ziegler [3].

TABLE 4. The Einstein function and its first five derivatives*

\begin{tabular}{|c|c|c|c|c|c|c|c|c|c|c|c|c|}
\hline \multirow{2}{*}{$X$} & \multicolumn{2}{|l|}{$E^{0}$} & \multicolumn{2}{|l|}{$E^{1}$} & \multicolumn{2}{|l|}{$E^{2}$} & \multicolumn{2}{|l|}{$E^{3}$} & \multicolumn{2}{|l|}{$E^{4}$} & \multicolumn{2}{|l|}{$E^{5}$} \\
\hline & Coef & $\operatorname{Exp}$ & Coef & $\operatorname{Exp}$ & Coef & $\operatorname{Exp}$ & Coef & Exp & Coef & $\operatorname{Exp}$ & Coef & $\operatorname{Exp}$ \\
\hline 1 & 9.20674 & - & -1.50948 & - & -1.21319 & & 8.19752 & - & 4.93166 & -2 & 53277 & -2 \\
\hline 2 & 7.24062 & - & -2.26657 & & -2.89466 & & 8.94460 & & -2.89868 & & .50503 & \\
\hline 3 & 4.96269 & -1 & -2.17428 & -1 & 3.97080 & & 4.43374 & - & -5.06061 & & 7.34212 & \\
\hline 4 & 3.04087 & - & -1.63391 & -1 & 6.13399 & & 2.48308 & & -3.00279 & & 2.62514 & \\
\hline 5 & 1.70742 & - & -1.04762 & - & 5.29512 & & -1.54893 & & -7.59110 & - & 1.66883 & \\
\hline 6 & 8.96791 & - & -6.02318 & -2 & 3.59185 & - & -1.68202 & -2 & 3.02914 & -3 & 5.38369 & -3 \\
\hline 7 & 4.47638 & - & -3.20559 & - & 2103 & & -1.22491 & & 38 & & 4766 & -4 \\
\hline 8 & 2.14840 & - & -1.61274 & - & 1.14494 & & -7.45505 & & 15200 & & -1.55181 & - \\
\hline 9 & 866 & - & -7.77921 & & 0800 & & -4.08606 & & 1503 & & -1.39754 & - \\
\hline 10 & 4.54041 & - & -3.63274 & - & 2.81612 & & -2.09076 & & 1.45695 & - & -9.15259 & - \\
\hline 11 & 2.02097 & - & -1.65359 & - & 1.31966 & - & -1.01920 & - & 7.52 & -4 & -5.19 & -4 \\
\hline 12 & 8.84777 & - & -7.37325 & & 9 & & -4 & & & & 70560 & -4 \\
\hline 13 & 3.81997 & - & 3.23230 & & 3985 & & -2.19263 & & 5 & & -1.33395 & -4 \\
\hline 14 & 1.62980 & - & -1.39697 & - & 1.18078 & & -9.81221 & - & 9 & & .32020 & - \\
\hline 15 & 6.88281 & - & -5.96510 & - & 5.10858 & - & -4.31325 & - & 3.57911 & 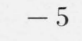 & -2.90616 & - \\
\hline 16 & 8090 & - & -2.52079 & - & 8 & - & -1.8 & - & 0 & -5 & -1 . & - \\
\hline 17 & 1.19644 & & -1.05568 & & 23206 & & -7.99009 & & 91 & & -5.75454 & - \\
\hline 18 & 4.93451 & - & 4.38623 & - & 86842 & & -3.38106 & & 16 & & -2.49772 & - \\
\hline 19 & 2.02261 & - & -1.80970 & - & 00 & - & -1.41751 & - & 1. & - & 07013 & - \\
\hline 20 & 8.24461 & - & -7.42015 & -7 & 6.63691 & - & -5.89490 & -7 & 5.19411 & -7 & -4.53454 & -7 \\
\hline 21 & 3.34391 & -7 & -3.02544 & -7 & 214 & -7 & -2.43400 & -7 & 3 & -7 & -1.9 & -7 \\
\hline 22 & 1.35010 & & 1.22737 & & & & -9.986 & & & & & \\
\hline 23 & 5.42853 & -8 & -4.95649 & -8 & 4.50497 & - & -4.07397 & - & 3.66 & - & -3.27354 & - \\
\hline 24 & 2.17448 & - & -1.99327 & -8 & 1.81961 & - & -1.65351 & - & 1.49495 & - & -1.34395 & - \\
\hline 25 & 8.67997 & & -7.98557 & & 7.31895 & & -6.68010 & & 6.06903 & & -5.48574 & - \\
\hline 26 & 3.45374 & - & -3.18807 & -9 & 2.93262 & -9 & -2.68738 & -9 & 2.45236 & -9 & -2.22756 & -9 \\
\hline 27 & 1.37018 & - & -1.26868 & & 1.17095 & & -1.07697 & & & & -9.00294 & -10 \\
\hline 28 & 5.42089 & -10 & -5.03368 & -10 & 4.66031 & -10 & -4.30076 & & 3.95504 & & -3.62315 & -10 \\
\hline 29 & 2.13922 & -10 & -1.99169 & -10 & 1.84925 & -1 & -1.71189 & -10 & 1.57962 & -10 & -1.45243 & -10 \\
\hline 30 & 8.42186 & -11 & -7.86040 & -11 & 7.31766 & -11 & -6.79363 & -11 & 6.28832 & -11 & -5.80173 & -11 \\
\hline
\end{tabular}

* The superscript numerals indicate the order of differentiation.

The Grüneisen function and its first five derivatives are computed using eqs (3), (13), (14), (15), (16), and (17), respectively. The results are given in table 5. The Grüneisen function expressed 
TABLE 5. The Grüneisen function and its first five derivatives*

\begin{tabular}{|c|c|c|c|c|c|c|c|c|c|c|c|c|c|c|}
\hline \multirow{2}{*}{$X$} & \multicolumn{2}{|l|}{$J_{5}^{* *}$} & \multicolumn{2}{|l|}{$G^{0}$} & \multicolumn{2}{|l|}{$G^{1}$} & \multicolumn{2}{|l|}{$G^{2}$} & \multicolumn{2}{|l|}{$G^{3}$} & \multicolumn{2}{|l|}{$G^{4}$} & \multicolumn{2}{|l|}{$G^{5}$} \\
\hline & Coef & Exp & Coef & $\operatorname{Exp}$ & Coef & $\operatorname{Exp}$ & Coef & $\operatorname{Exp}$ & Coef & Exp & Coef & $\operatorname{Exp}$ & Coef & $\operatorname{Exp}$ \\
\hline 1 & 2.36616 & -1 & 9.46463 & -1 & -1.03156 & -1 & -8.80120 & -2 & 4.27961 & -2 & 2.83281 & -2 & -2.93585 & -2 \\
\hline 2 & 3.22929 & 0 & 8.07321 & -1 & -1.66518 & -1 & -3.70190 & -2 & 5.31638 & -2 & -7.18128 & -3 & -2.92485 & -2 \\
\hline 3 & 1.27705 & +1 & 6.30643 & -1 & -1.79165 & -1 & 8.70489 & -3 & 3.55342 & -2 & -2.37967 & -2 & -4.01706 & -3 \\
\hline 4 & 2.94882 & +1 & 4.60754 & -1 & -1.56667 & -1 & 3.24428 & -2 & 1.26758 & -2 & -1.96995 & -2 & 9.37115 & -3 \\
\hline 5 & 5.02629 & +1 & 3.21682 & -1 & -1.20752 & -1 & 3.69424 & -2 & -1.96992 & -3 & -9.63355 & -3 & 9.34080 & -3 \\
\hline 6 & 7.08729 & +1 & 2.18744 & -1 & -8.60433 & -2 & 3.15482 & -2 & -7.60252 & -3 & -2.34386 & -3 & 5.14457 & -3 \\
\hline 7 & 8.83248 & +1 & 1.47147 & -1 & -5.85047 & -2 & 2.34714 & -2 & -7.99817 & -3 & 9.98689 & -4 & 1.83229 & -3 \\
\hline 8 & 1.01477 & +2 & 9.90984 & -2 & -3.88072 & -2 & 1.61908 & -2 & -6.41840 & -3 & 1.88858 & -3 & 1.87425 & -4 \\
\hline 9 & 1.10546 & +2 & 6.73958 & -2 & -2.55098 & -2 & 1.07147 & -2 & -4.56180 & -3 & 1.73204 & -3 & -3.77359 & -4 \\
\hline 10 & 1.16379 & +2 & 4.65517 & -2 & -1.68045 & -2 & 6.94916 & -3 & -3.04305 & -3 & 1.29383 & -3 & -4.52284 & -4 \\
\hline 11 & 1.19927 & +2 & 3.27647 & -2 & -1.11795 & -2 & 4.48030 & -3 & -1.96393 & -3 & 8.79152 & -4 & -3.65823 & -4 \\
\hline 12 & 1.21988 & +2 & 2.35317 & -2 & -7.54897 & -3 & 2.89963 & -3 & -1.24909 & -3 & 5.68866 & -4 & -2.56320 & -4 \\
\hline 13 & 1.23141 & +2 & 1.72460 & -2 & -5.18892 & -3 & 1.89628 & -3 & -7.92444 & -4 & 3.59235 & -4 & -1.67509 & -4 \\
\hline 14 & 1.23765 & +2 & 1.28868 & -2 & -3.63538 & -3 & 1.25844 & -3 & -5.05593 & -4 & 2.24762 & -4 & -1.05627 & -4 \\
\hline 15 & 1.24093 & +2 & 9.80489 & -3 & -2.59628 & -3 & 8.49521 & -4 & -3.26185 & -4 & 1.40718 & -4 & -6.55052 & -5 \\
\hline 16 & 1.24262 & +2 & 7.58434 & -3 & -1.88888 & -3 & 5.83974 & -4 & -2.13532 & -4 & 8.87501 & -5 & -4.04363 & -5 \\
\hline 17 & 1.24347 & +2 & 5.95523 & -3 & -1.39842 & -3 & 4.08815 & -4 & -1.42115 & -4 & 5.66380 & -5 & -2.50459 & -5 \\
\hline 18 & 1.24388 & +2 & 4.73969 & -3 & -1.05217 & -3 & 2.91294 & -4 & -9.62384 & -5 & 3.66747 & -5 & -1.56501 & -5 \\
\hline 19 & 1.24409 & +2 & 3.81853 & -3 & -8.03475 & -4 & 2.11060 & -4 & -6.63120 & -5 & 2.41323 & -5 & -9.90029 & -6 \\
\hline 20 & 1.24418 & +2 & 3.11045 & -3 & -6.21925 & -4 & 1.55333 & -4 & -4.64671 & -5 & 1.61456 & -5 & -6.35436 & -6 \\
\hline 21 & 1.24422 & +2 & 2.55907 & -3 & -4.87378 & -4 & 1.15985 & -4 & -3.30867 & -5 & 1.09825 & -5 & -4.14266 & -6 \\
\hline 22 & 1.24424 & +2 & 2.12459 & -3 & -3.86265 & -4 & 8.77651 & -5 & -2.39157 & -5 & 7.59140 & -6 & -2.74428 & -6 \\
\hline 23 & 1.24425 & +2 & 1.77851 & -3 & -3.09297 & -4 & 6.72298 & -5 & -1.75304 & -5 & 5.32824 & -6 & -1.84693 & -6 \\
\hline 24 & 1.24425 & +2 & 1.50011 & -3 & -2.50015 & -4 & 5.20831 & -5 & -1.30177 & -5 & 3.79408 & -6 & -1.26220 & -6 \\
\hline 25 & 1.24425 & +2 & 1.27411 & -3 & -2.03856 & -4 & 4.07700 & -5 & -9.78362 & -6 & 2.73834 & -6 & -8.75299 & -7 \\
\hline 26 & 1.24425 & +2 & 1.08912 & -3 & -1.67556 & -4 & 3.22219 & -5 & -7.43537 & -6 & 2.00142 & -6 & -6.15443 & -7 \\
\hline 27 & 1.24425 & +2 & 9.36511 & -4 & -1.38742 & -4 & 2.56928 & -5 & -5.70934 & -6 & 1.48004 & -6 & -4.38384 & -7 \\
\hline 28 & 1.24425 & +2 & 8.09722 & -4 & -1.15674 & -4 & 2.06561 & -5 & -4.42624 & -6 & 1.10650 & -6 & -3.16086 & -7 \\
\hline 29 & 1.24425 & +2 & 7.03681 & -4 & -9.70594 & -5 & 1.67344 & -5 & -3.46225 & -6 & 8.35693 & -7 & -2.30514 & -7 \\
\hline 30 & 1.24425 & +2 & 6.14445 & -4 & -8.19260 & -5 & 1.36543 & -5 & -2.73085 & -6 & 6.37190 & -7 & -1.69909 & -7 \\
\hline
\end{tabular}

$*$ The superscript numerals indicate the order of differentiation.
$* *$ For $x=\infty, J_{5}=124.4313[2]$. 
by eq (3) may be written as

where

$$
G^{0}(x)=\left(\frac{4}{x^{4}}\right) J_{5}
$$

$$
J_{5}=\int_{0}^{x} \frac{\zeta^{5} d \zeta}{\left(e^{\zeta}-1\right)\left(1-e^{-\zeta}\right)}
$$

There is no known closed form for the above integration, therefore numerical integration techniques are employed. Various values for the increment in numerical integration are tried to determine the convergence of the results. Values 0.002 and 0.0002 yielded a difference of 0.00042 percent for $x=1$, and 0.0048 percent for $x=30 \mathrm{in} J_{5}$. In the literature, Rogers and Powell [4] have computed the integral $J_{5}$ utilizing series expansion approximation. Their results correspond exactly to those obtained using a value of 0.002 for the increment. Since the accuracy of the approximation of numerical integration increases with decreasing increment size, a value of 0.0002 is used in the final computations. Thus, it is expected that the present results of $J_{5}$ and hence the Grüneisen function are more accurate than those given by Rogers and Powell.

It may be noted that the only numerical method employed in the present computations is that for the evaluation of $J_{5}$, the integral appearing in the Grüneisen function. All other computations including those for the first five derivatives of the Grüneisen and Einstein functions represent mathematically closed forms and thus are not subject to errors common to numerical differentiation.

\section{Summary}

The Grüneisen and Einstein functions appear in the mathematical expressions describing a number of thermodynamic and transport properties of matter. In order to improve the interpretation of experimental results and extend the application of the theories regarding these properties, a knowledge of the temperature derivatives of the above functions are needed. In the present study, mathematical expressions in closed form are derived for the derivatives of the Grüneisen and Einstein functions. Also, the first five derivatives of both functions are computed and presented in tabular form. Since all the derivatives are obtained from mathematical relations of closed form, no errors inherent to numerical differentiation are introduced.

The author extends his appreciation to C. W. Beckett for suggesting the undertaking of this study.

\section{References}

[1] Gerritsen, A. N., Metallic Conductivity, in Handb. der Physik, Vol. 19, p. 171 (Springer, Berlin, 1956).

[2] Gradshteyn, I. S., and Ryzhik, I. M., Table of Integrals, Series and Products, p. 330 (Academic Press, New York, 1965).

[3] Hilsenrath, J., and Ziegler, G. G., Tables of Einstein Functions, Nat. Bur. Stand. (U.S.), Monogr. 49, 258 pages (1962).

[4] Rogers, W. M., and Powell, R. L., Tables of Transport Integrals, Nat. Bur. Stand. (U.S.), Circ. 595, 46 pages (1958).

(Paper 74B3-328) 